lower proportion of lymphocytes $(70 \%$ vs $93 \%, \mathrm{p}=0.0085)$ and a higher proportion of neutrophils $(\mathrm{p}=0.033)$. An elevated $\mathrm{IgG}$ index occurred in fewer earlier-onset disease patients than in the later-onset group ( $35 \%$ vs $68 \%, p=0.031)$. An activation of the innate rather than the adaptive immune system in the earlier stages of MS is suggested or an immature immune response. (Chabas D, Ness J, Belman A, et al, for the US Network of Pediatric MS Centers of Excellence. Neurology Feb 2, 2010;74(5):399-405). (Reprints: Dr Dorothy Chabas, UCSF Regional Pediatric MS Center, 350 Parnasus Avenue, Suite 908, San Francisco, CA 84117). E-mail: dchabas@gmail.com).

COMMENT. The CSF profile in pediatric MS is dependent on age of onset, and the age-related variations are important in diagnosis. Pohl D et al (Neurology 2004;63:1966-7) reported the CSF characteristics of 136 childhood-onset (<16 years) cases of MS. CSF pleocytosis occurred in $66 \%$, and oligoclonal IgG in $92 \%$.

\title{
ACUTE DISSEMINATED ENCEPHALOMYELITIS AND MULTIPLE SCLEROSIS COMPARED
}

Clinical characteristics at presentation and follow-up of a cohort of 13 cases (age range 5-67, median 43 years) of perivenous demyelination were compared with a cohort of 91 cases (age range 10-69, median 39 years) of confluent demyelination (76 [84\%] cases of confirmed multiple sclerosis) in a study at the Mayo Clinic, Rochester, MN. Clinical criteria for ADEM were applied to both groups to assess their ability to distinguish ADEM from MS, as determined pathologically. Only two patients with perivenous demyelination were pediatric (aged 5 and 17 years). All but one patient with perivenous demyelination had a monophasic course, whereas two of three with both periand confluent-demyelination had a relapsing course. The perivenous cohort was more likely than the confluent demyelination cohort to present with encephalopathy, depressed consciousness, headache, meningismus, CSF pleocytosis or multifocal enhancing MRI lesions. Pathological studies showed a distinct pattern of cortical microglial activation and aggregation without associated cortical demyelination in six perivenous demyelination patients, all of whom had encephalopathy. This cortical pathology was not found in the confluent demyelination cohort. Clinical citeria were $80 \%$ sensitive and $91 \%$ specific for pathologically defined ADEM. Perivenous demyelination has a meningoencephalopathic presentation and a monophasic course. The co-occurrence of perivenous and confluent demyelination in some patients suggests an overlap between ADEM and MS and misclassification. (Young NP, Weinshenker BG, Parisi JE, et al. Perivenous demyelination: association with clinically defined acute disseminated encephalomyelitis and comparison with pathologically confirmed multiple sclerosis. Brain Feb 2010;133:333-348). (Respond: Claudia F Luchinetti MD, Department of Neurology, Mayo Clinic College of Medicine, $2001^{\text {st }}$ Street SW, Rochester, MN 55905. E-mail: luchinetti.claudia@mayo.edu).

COMMENT. Lassmann H (Brain Feb 2010;133:317-318) comments that the study shows the clinical criteria for the differentiation of ADEM and MS are imperfect, with considerable overlap in clinical presentation. Pathological confirmation of the clinical diagnosis of ADEM may help to refine ADEM clinical criteria. 